\title{
The Value of Certified Pre-Owned Vehicles for Lexus of Edmonton
}

Trisha L Kasawski, Sunaina Singh, Kanwarbir Bhullar, Gurman Sidhu, Gurman

\begin{abstract}
Our objective was to discover productive improvements to Lexus of Edmonton's (LoE) current Certified Pre-Owned (CPO) Vehicle practices by determining if CPO vehicles added value, and if so, what value was added. By utilizing multiple methods, we discovered areas in the used vehicle sector that Lexus of Edmonton could improve on. In order to conduct our research, we conducted five in-depth interviews, reviewed scholarly article evaluations, had meetings with the representatives of Lexus of Edmonton, and conducted surveys using paper, email, and social media tools such as Google surveys, Twitter and Facebook. These methods allowed our group to utilize statistical analysis to outline the importance of relationships between variables with the statistical software, SPSS. With the information received from statistical analysis, we believe that Lexus of Edmonton can succeed with the sale of CPO vehicles by following our recommendations: certification education, appropriate pricing of used vehicles, building trust, valueadded certification benefits based on willingness to spend and targeted demographics, as well as further research.
\end{abstract}

Keywords: customer service, automobile industry, pre-owned, used dealership, luxury dealership, Certified Preowned vehicles, warranty, marketing, advertising, gender, vehicle financing, used car certification, young consumer finances, Lexus, brand awareness, brand perception

\section{Problem Definition}

Vol. 4(1) | DOI: https://doi.org/10.31542/muse.v4i1.864 MacEwan University Student eJournal

(C) 2020 under CC BY-NC I ISSN 2369-5617 


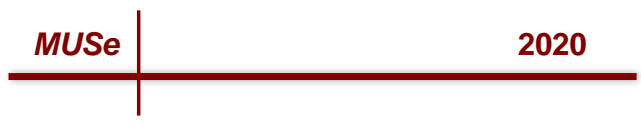

LoE has continued to lead the market in the industry of automobiles within Edmonton. With a manufacturer's certified pre-wwned process already in place for all used vehicles, LoE is considering remodeling the certification of pre-owned vehicles to cover all used vehicles, seeking to add what is most important to customers. When interviewing LoE, the management problem was whether or not certified pre-owned vehicles added value to luxury dealerships. The marketing research problem was to determine how CPO vehicles benefit consumer needs through awareness, finances, and overall standing in our present-day economy. In redefining the marketing research problem, we want to determine what specific value meant to customers and if the value was an investment worth making for Lexus of Edmonton.

Tasks completed included five in-depth interviews with potential customers, an analysis of over 20 academic sources, two white papers, a paper including vital statistics, research on the value of marketing research, and an in-depth interview with LoE. Further online questionnaires were conducted after examining qualitative research findings. We surveyed 128 respondents who gave us relevant information and statistics that supported our research findings. 21 of those respondents were over the age of 25; they were removed from the SPSS statistical analysis, as they are not relevant to our targeted market of ages 25 and under.

Our intent is to gain a greater understanding if and how CPO vehicles benefit consumer needs through finances, awareness, and overall standing in today's society. We were provided with information to create marketing strategies that will be used by car dealerships to promote and increase the success of preowned inventory. We can use this information to provide constructive feedback to Lexus of Edmonton on what is valued in certified pre-owned vehicles and if it is worth pursuing.

\section{Analytical Framework of the Marketing Research Problem}


Based on our literature review and qualitative research analysis, we were able to summarize the two problems we

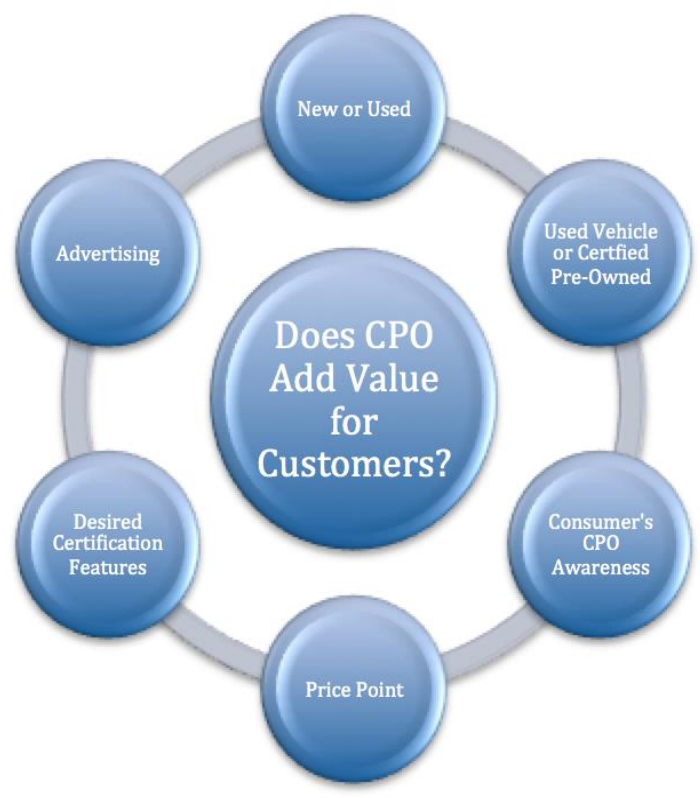

presented: the perspective of the marketing researcher, and the perspective of management. The marketing research problem was to see if and how CPO vehicles benefit consumer needs through awareness, finances, and overall standing in our present-day economy. The management problem was if it added value to the dealership and if it is an investment worth making.

Figure 1: Does CPO Add Value for Customers 


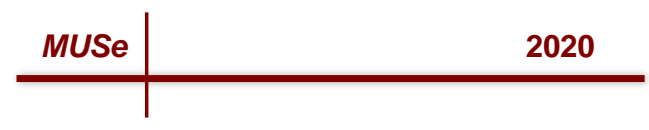

In terms of redefining the marketing research problem, we realized the importance of customer awareness, affordability, and desired certification features for our targeted market. The four main issues we researched are as follows:

- Current awareness of Lexus and Certified Pre-owned vehicles

- Customer service

- Overall marketing strategies of Certified Pre-owned vehicles in luxury dealerships as a whole

- Financing of Certified Pre-Owned vehicles

Research Questions and Hypothesis

Question 1: What does certification mean to used-car customers?

Hypothesis: Based on the in-depth interviews we conducted in our initial report, we hypothesize the majority of consumers have varied knowledge on what certification means (Jones, 1997; Lewis, 2015; Nemtuda, 2013; Peters, 2002; Sawyers \& Treece, 2016; Sultan, 2010).

Question 2: How likely will consumers purchase a pre-owned vehicle over a new vehicle?

Hypothesis: We hypothesize that consumers who identify as female are the most likely to purchase a used car over a brandnew vehicle, based off in-depth interviews.

Question 3: Do consumers believe there are benefits of a Certified Pre-Owned Vehicle opposed to a regular pre-owned vehicle?

Hypothesis: Based on our in-depth interviews, we discovered the term "certified" suggested supremacy over non-CPO vehicles, regardless of what is known about certification. We hypothesize that the majority of the targeted market are willing to purchase a CPO vehicle over a regular pre-owned vehicle, regardless of their level of certification knowledge 
(Jones, 1997; Lewis, 2015; Nemtuda, 2013; Peters, 2002; Sawyers, 2010; Sawyers \& Treece, 2016; Sultan, 2010).

Question 4: How much are consumers willing to spend on a purchase of a certified pre-owned vehicle?

Hypothesis: Based on our exploratory research, we predict consumers are willing to spend an average of $\$ 15,000$ $\$ 20,000$ on CPO vehicles. We hypothesize that students will purchase used vehicles that are mid-priced (Busse, Knittel \& Zettelmeyer, 2013; Fan \& Burton, 2005; McDonald, 2012; Peterson \& Schneider, 2014; Sawyers, 2010; Shipp, 2013; Strautmanis \& Hermanis, 2013).

Question 5: How much would consumers pay overall for a Certified Pre-Owned including their preferred features? Hypothesis: After conducting in-depth interviews and exploratory research, we hypothesize that consumers will pay higher amounts for CPO vehicles that their preferred features. Additionally, we hypothesize consumers will pay more for four features, based on the in-depth interviews: a vehicle with less than 80,000 kilometers, one-year end to end warranty, CarProof verification, and one that was made within the last 7 years (Busse, Knittel \& Zettelmeyer, 2013; Cristian \& Nicoleta, 2011; Mcdonald, 2012; Peters, 2002; Peterson \& Schneider, 2014; Sawyers, 2010; Shipp, 2013; Strautmanis \& Hermanis, 2013).

Question 6: Will gender affect preference in paying more for used over certified pre-owned vehicles if the CPO costs more? Hypothesis: Through secondary and exploratory research, we predict that gender will directly affect willingness to pay more for CPO. We hypothesize that males are willing to spend more money than females when purchasing a CPO over a used one (Lacetera, Pope, \& Sydnor, 2012; Shipp, 2013). 


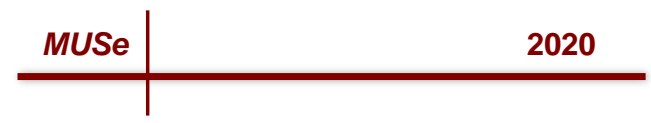

Question 7: What would consumers like to see when advertising certified pre-owned vehicles to their customers? Hypothesis: Secondary research revealed different advertising methods appealed to raising brand awareness (Barkholz, 2015; Chappell, 2015; Hsiao, Hsu, \& Fang, 2014; Mattera, Baena, \& Cerviño, 2012; Zarantonello, Schmitt \& Jedidi, 2014). Based on the in-depth interviews we conducted, we hypothesize that consumers prefer higher certification points advertised, even if they do not know what the points cover.

Question 8: Do consumers who trust Lexus of Edmonton value certified and non-certified pre-owned vehicles equally? Hypothesis: Exploratory research predicts when trust is low, there may be a difference between value of certified versus noncertified (Ažman \& Gomišček, 2014; Confente \& Russo, 2015). After analysis of in-depth interviews, we found respondents were concerned with the low level of trust they had towards dealerships due to the fact that consumers feel like dealerships only want their money.

Question 9: Does trust in Lexus of Edmonton increase consumers' willingness to pay more for a Certified Pre-owned Vehicle?

Hypothesis: We hypothesize where high levels of trust exist, consumers are willing to spend more on certified vehicles (Ažman \& Gomišček, 2014; Confente \& Russo, 2015). Our indepth interviews suggested that trust is important when customers make final decision in purchasing a used CPO vehicle.

Question 10: Do consumers trust Lexus of Edmonton in selling them a certified pre-owned dealership?

Hypothesis: Based on our secondary research, the correlation between the satisfaction level and trust rating is positive; satisfied customers tend to be more trusting (Ažman \& Gomišček, 2014; Confente \& Russo, 2015). We predict the 
majority of consumers will purchase a certified pre-owned vehicle from a dealership they trust.

\section{Descriptive Research Design}

\section{Data Collection}

We first identified the problem Lexus of Edmonton presented by interviewing the Dealer Principal and General Manager. With the use of secondary research, we completed a literature review to help support the research problem identified. Five indepth interviews were held in order to formulate an analysis, identifying areas we believe would assist Lexus of Edmonton. We developed an anonymous survey to collect our data, totaling 128 responses. The majority of responses were gathered online through the shared questionnaire via email, Facebook, Twitter and Instagram, with the majority of responses coming from Facebook. Based on the targeted market segment, we believed Facebook would be the most effective method in data collection. Additional responses came from a class at MacEwan University who filled out a hard copy of the questionnaire. For the purpose of this study we used 107 of the respondents who identified as being of the age of 25 and under (the additional 21 were not in our targeted market). 
Table 1: Respondent Demographics

\begin{tabular}{|c|c|c|c|}
\hline Variables & \multicolumn{2}{|c|}{ \# of Respondents } & $\begin{array}{c}\% \text { of } \\
\text { Respondents }\end{array}$ \\
\hline \multicolumn{4}{|l|}{ Gender } \\
\hline Female & \multicolumn{2}{|c|}{42} & $39.3 \%$ \\
\hline Male & \multicolumn{2}{|c|}{61} & $57.0 \%$ \\
\hline $\begin{array}{l}\text { Prefer Not to } \\
\text { Answer }\end{array}$ & \multicolumn{2}{|c|}{4} & $3.7 \%$ \\
\hline \multirow[t]{2}{*}{ Total Responses } & \multicolumn{2}{|c|}{107} & $100.0 \%$ \\
\hline & \multicolumn{2}{|c|}{ Responses } & \multirow[b]{2}{*}{$\%$ Of Cases } \\
\hline $\begin{array}{l}\text { Current } \\
\text { Occupation }\end{array}$ & $\mathrm{N}$ & Percent & \\
\hline Full Time & 12 & $8.4 \%$ & $11.2 \%$ \\
\hline Unemployed & 2 & $61.5 \%$ & $82.2 \%$ \\
\hline Student & 88 & $1.4 \%$ & $1.9 \%$ \\
\hline Part Time & 41 & $28.7 \%$ & $38.3 \%$ \\
\hline $\begin{array}{l}\text { Total } \\
\text { Responses }\end{array}$ & 143 & $100.0 \%$ & $133.6 \%$ \\
\hline
\end{tabular}


Development, Pre-test and Execution of the Questionnaire We met on a bi-weekly basis in order to compile the questionnaire. Pre-testing was done with a university marketing class, presenting evaluations and recommendations, which assisted us in identifying slight issues with question comprehension. Following this analysis, we presented LoE with the questionnaire in order to receive insight to make additional minor adjustments where needed.

\section{Ethical Issues}

Respondents were presented with a consent form approved by the research ethics board, and were allowed to terminate the survey at anytime. Due to the anonymity of the online survey, there could be potential issues with complete honesty or falsification within the survey responses. As the survey was presented on a public domain, responses may be compromised based on what respondents value, or their intent as to why they chose to participate in the study. Overall, there were no ethical issues on the survey we conducted.

\section{Data Analysis and Results}

Plan of Data

We conducted four Analysis of Variance (ANOVA) tests, two multiple response cross- tabulations, three cross tabulations analysis of Chi-Square Tests, and one Pearson correlation analysis. We used univariate and bivariate techniques to further study key variables. ANOVA tests were conducted in order to compare the trust consumers have in the Lexus of Edmonton' pre-owned dealership, the value of Certified PreOwned over non-certified, consumer willingness to pay for a Certified Pre-owned vehicle, and level of satisfaction with Lexus of Edmonton. Cross-tabulations and multiple response cross-tabulations were selected to discover relationships between variables. We discovered relationships between customer's CPO preference and knowledge of what a CPO 


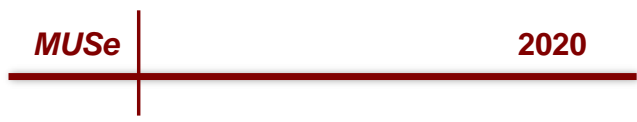

vehicle is, and the relationship between customer's likelihood to purchase used vehicles and current occupation.

We also performed bivariate analysis with cross-tabulations to determine the best advertising technique to engage the 25 and under market. Using Chi-Square tests, we were able to relate variables in order to support or negate our hypotheses. We recoded questions $1,4,5$ and 12 in order to ensure proper placement of the variables analyzed.

The goal of our marketing research was determining if CPO vehicles added value to Lexus of Edmonton, and if so, what consumers considered valuable. We discovered many positive correlations and information that assisted us in determining next steps for Lexus of Edmonton's Certified Pre-Owned vehicle market.

\section{Results}

Question 1: What does certification mean to used-car customers?

Using univariate analysis, Table 2 shows that $68.2 \%$ of those surveyed knew what a certified pre-owned vehicle was, while $31.8 \%$ did not. Preferences for what should be included in a certified pre-owned vehicle varied (Table 3 ), with "less than 80K kilometers" (17.4\%) leading, followed by "CarProof verification* includes accident report, recall, liens, etc." (14.9\%), and "made within the last 7 years" and "one year endto-end warranty" tied at $14.5 \%$ each.

Table 2: Customers' Knowledge of What a Certified Pre-owned Vehicle Is

\begin{tabular}{|l|c|c|}
\hline \multicolumn{1}{|c|}{ Variable } & \# of Respondents & \% of Respondents \\
\hline No & 34 & $31.8 \%$ \\
\hline Yes & 73 & $68.2 \%$ \\
\hline Total & 107 & $100.0 \%$ \\
\hline
\end{tabular}


Kasawski, Singh, Bhullar, Sidhu, Bath

Table 3: Customers' Preference as to what should be Included in a Certified

Pre-owned Vehicle

\begin{tabular}{|c|c|c|}
\hline Variable & $\begin{array}{c}\text { \# of } \\
\text { Respondents }\end{array}$ & $\%$ of Respondents \\
\hline $\begin{array}{l}4 \text { months/40K km } \\
\text { Powertrain and Roadside } \\
\text { Assistance coverage }\end{array}$ & 52 & $10.0 \%$ \\
\hline $\begin{array}{l}\text { CarProof verification* } \\
\text { includes accident report, } \\
\text { recall, liens, etc. }\end{array}$ & 77 & $14.9 \%$ \\
\hline $\begin{array}{l}\text { Complimentary oil \& filter } \\
\text { service }\end{array}$ & 49 & $9.5 \%$ \\
\hline $\begin{array}{l}\text { Enrollment with dealership } \\
\text { exclusives, including private } \\
\text { events, free rentals, and VIP } \\
\text { services }\end{array}$ & 32 & $6.2 \%$ \\
\hline $\begin{array}{l}\text { Five/Ten (5-10) day/1500 km } \\
\text { (whichever comes first) grace } \\
\text { period for swap or trade }\end{array}$ & 45 & $8.7 \%$ \\
\hline Full tank of gas & 22 & $4.2 \%$ \\
\hline Made within the last 7 years & 75 & $14.5 \%$ \\
\hline $\begin{array}{l}\text { One year end-to-end } \\
\text { warranty }\end{array}$ & 75 & $14.5 \%$ \\
\hline Less than $110 \mathrm{~K}$ kilometers & 1 & $0.2 \%$ \\
\hline Less than $80 \mathrm{~K}$ kilometers & 90 & $17.4 \%$ \\
\hline Total & 518 & $100.0 \%$ \\
\hline
\end{tabular}

We observed through multiple response cross-tabulation (Table 4) that regardless of the level of certification knowledge, 


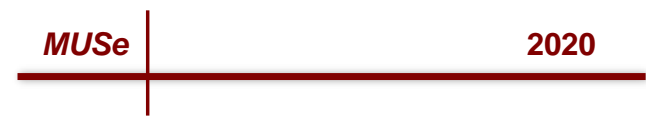

consumers preferred similar variables, differing in order of value. Consumers who have no certification knowledge (31.9\%) prefer less than $80 \mathrm{~K}(5.4 \%)$, followed by made within the last 7 years (5.2\%), CarProof verification (4.6\%), and one-year endto-end warranty (4.4\%). Consumers who have certification knowledge (68.1\%) prefer less than $80 \mathrm{~K}(12.0 \%)$, followed by CarProof verification (10.2\%), one-year end-to-end warranty $(10.0 \%)$, and made within the last 7 years $(9.3 \%)$. Based on the above information, we fail to reject the Ho.

Table 4: Multiple Response Cross Tabulation Between Customer's CPO Preference and Knowledge of What a CPO Vehicle is

\begin{tabular}{|c|c|c|c|c|c|}
\hline & & & Certification & wedge & \\
\hline & & & No & Yes & Total \\
\hline \multirow{30}{*}{$\begin{array}{l}\text { CPO } \\
\text { Preferred } \\
\text { Options }\end{array}$} & \multirow{3}{*}{$\begin{array}{l}4 \text { months/40K km Powertrain } \\
\text { and Roadside Assistance } \\
\text { coverage }\end{array}$} & Count & 17 & 35 & 52 \\
\hline & & 96 within soptions & $327 \%$ & $67.3 \%$ & \\
\hline & & $\%$ \& Total & 3.396 & $6.8 \%$ & $10.0 \%$ \\
\hline & \multirow{3}{*}{$\begin{array}{l}\text { CarProof verification" includos } \\
\text { acadent report, recall, liens, etc }\end{array}$} & Count & 24 & 53 & 77 \\
\hline & & \% within Soptions & $31.2 \%$ & $68.8 \%$ & \\
\hline & & \% of Total & 4. $6 \%$ & $10.2 \%$ & $14.9 \%$ \\
\hline & \multirow{3}{*}{$\begin{array}{l}\text { Complimentary oil \& fitter } \\
\text { senice }\end{array}$} & Count & 10 & 39 & 49 \\
\hline & & $\%$ within Soptions & $20.4 \%$ & $79.6 \%$ & \\
\hline & & $\%$ of Total & $1.9 \%$ & $7.5 \%$ & $9.5 \%$ \\
\hline & \multirow{3}{*}{$\begin{array}{l}\text { Enroliment with dealership } \\
\text { exclusives, incluang private } \\
\text { events, free rentals, and VIP } \\
\text { services }\end{array}$} & Count & 10 & 22 & 32 \\
\hline & & $\%$ within Soptions & $31.3 \%$ & $688 \%$ & \\
\hline & & \% of Total & 1.99 & $4.2 \%$ & $6.2 \%$ \\
\hline & \multirow{3}{*}{$\begin{array}{l}\text { Five/Ten }(5-10) \text { dayl } 1500 \mathrm{~km} \\
\text { (Whichever comes first) grace } \\
\text { period for swap or trade }\end{array}$} & Count & 19 & 26 & 45 \\
\hline & & \% within soptions & 42.29 & 57.896 & \\
\hline & & \% of Total & $3.7 \%$ & $5.0 \%$ & $8.7 \%$ \\
\hline & \multirow[t]{3}{*}{ Full tank of gas } & Count & 7 & 15 & 22 \\
\hline & & $\%$ within Soptions & $31.8 \%$ & $68.2 \%$ & \\
\hline & & $\%$ of Tot al & $1.4 \%$ & $29 \%$ & $4.2 \%$ \\
\hline & \multirow[t]{3}{*}{ M ade within the last 7 years } & Count & 27 & 48 & 75 \\
\hline & & 96 within soptions & $36.0 \%$ & 6.096 & \\
\hline & & $\% \propto \alpha$ Total & $5.2 \%$ & $2.3 \%$ & $14.5 \%$ \\
\hline & \multirow[t]{3}{*}{ One year end-to-end warranty } & Count & 23 & 52 & 75 \\
\hline & & \% within Soptions & $30.7 \%$ & $69.3 \%$ & \\
\hline & & $\% \propto$ Total & 4. $4 \%$ & $10.0 \%$ & $14.5 \%$ \\
\hline & \multirow[t]{3}{*}{ Less than $110 \mathrm{~K}$ viometers } & Count & o & 1 & 1 \\
\hline & & $\%$ within soptions & $0.0 \%$ & $100.0 \%$ & \\
\hline & & \% a Total & $0.0 \%$ & $0.2 \%$ & $0.2 \%$ \\
\hline & \multirow[t]{3}{*}{ Less than Bok kilometers } & Count & 28 & 62 & 90 \\
\hline & & $\%$ within soptions & 31. 1\% & $68.9 \%$ & \\
\hline & & $\%$ of Tolal & $5.4 \%$ & $120 \%$ & $17.4 \%$ \\
\hline \multirow[t]{2}{*}{ Total } & & Count & 165 & 353 & 518 \\
\hline & & S of Tolal & $31.9 \%$ & $681 \%$ & $100.0 \%$ \\
\hline
\end{tabular}


Question 2: How likely will consumers purchase a pre-owned vehicle over a new vehicle?

Table 5 shows univariate analysis, revealing that $48.6 \%$ of respondents are "Very Likely" or "Likely" to purchase a certified-vehicle over a non-certified vehicle, whereas as $17.8 \%$ were "Unlikely" or "Very Unlikely".

Table 5: Customers Likelihood of Purchasing a Pre-owned Vehicle Over a New Vehicle

\begin{tabular}{|l|c|c|}
\hline \multicolumn{1}{|c|}{ Variable } & \# of Respondents & \% of Respondents \\
\hline Very Likely & 21 & $19.6 \%$ \\
\hline Likely & 31 & $29.0 \%$ \\
\hline Somewhat Likely & 30 & $28.0 \%$ \\
\hline Unlikely & 8 & $7.5 \%$ \\
\hline Very Unlikely & 11 & $10.3 \%$ \\
\hline Total & 101 & $94.4 \%$ \\
\hline
\end{tabular}

Based on Table 6 and 7 ANOVA results with 1 being very likely and 5 being very unlikely, females are more likely to purchase a pre-owned vehicle over males. Therefore, we fail to reject the Ho. 


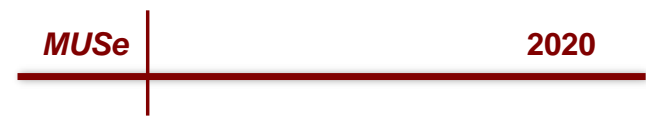

Table 6: ANOVA Between Customer's Likelihood to Purchase a Used Vehicle and Gender

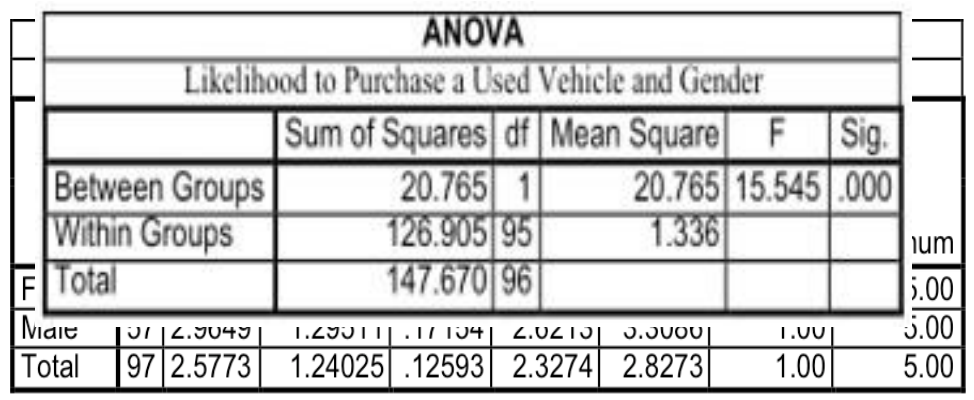

Table 7: ANOVA

Question 3: Do consumers believe there are benefits of a Certified Pre-Owned Vehicle, opposed to a regular pre-owned vehicle?

Through univariate analysis (Table 8 ), we found that $82.2 \%$ of respondents are willing to purchase a CPO over a non-CPO vehicle.

Table 8: Customers Willingness to Purchase a Certified Pre-Owned Vehicle Over a Non-Certified Pre-Owned Vehicle

\begin{tabular}{|l|c|c|}
\hline \multicolumn{1}{|c|}{ Variable } & \# of Respondents & \% of Respondents \\
\hline No & 19 & $17.8 \%$ \\
\hline Yes & 88 & $82.2 \%$ \\
\hline
\end{tabular}


We used cross-tabulation (Table 9) and chi-square (Table 10) to explore the relationship between variables. We concluded there is a positive correlation between consumers who have certification knowledge and willingness to purchase a CPO over a non-CPO (89.0\%). While smaller, there is still a positive correlation between consumers who have no certification knowledge and willingness to purchase a CPO over a non-CPO $(67.6 \%)$. There is a relation between these two variables. Therefore, we fail to reject the Ho.

Table 9: Cross Tabulation Between Consumers Certification Knowledge and Willingness to Purchase CPO Over Non-CPO Vehicles

\begin{tabular}{|c|c|c|c|c|c|}
\hline & & & \multicolumn{2}{|c|}{$\begin{array}{c}\text { Willingness to purchase CPO } \\
\text { over non-CPO }\end{array}$} & \multirow[b]{2}{*}{ Total } \\
\hline & & & No & Yes & \\
\hline \multirow{6}{*}{$\begin{array}{l}\text { Certification } \\
\text { Knowledge }\end{array}$} & \multirow[t]{3}{*}{ No } & Count & 11 & 23 & 34 \\
\hline & & Expected Count & 6.0 & 28.0 & 34.0 \\
\hline & & $\begin{array}{l}\% \text { Certification } \\
\text { Knowledge }\end{array}$ & $32.4 \%$ & $67.6 \%$ & $100.0 \%$ \\
\hline & \multirow[t]{3}{*}{ Yes } & Count & 8 & 65 & 73 \\
\hline & & Expected Count & 13.0 & 60.0 & 73.0 \\
\hline & & $\begin{array}{l}\% \text { Certification } \\
\text { Knowledge }\end{array}$ & $11.0 \%$ & $89.0 \%$ & $100.0 \%$ \\
\hline \multirow{3}{*}{\multicolumn{2}{|c|}{ Total }} & Count & 19 & 88 & 107 \\
\hline & & Expected Count & 19.0 & 88.0 & 107.0 \\
\hline & & $\begin{array}{l}\% \text { Certification } \\
\text { Knowledge }\end{array}$ & $17.8 \%$ & $82.2 \%$ & $100.0 \%$ \\
\hline
\end{tabular}




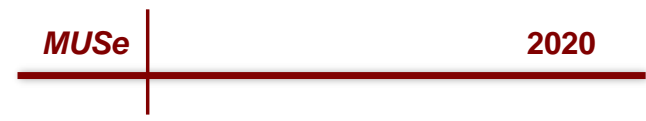

Table 10: Chi-Square Test

\begin{tabular}{|c|c|c|c|}
\hline & Value & $d f$ & Asymptotic Significance (2-sided) \\
\hline Pearson Chi-Square & $7.270^{\mathrm{d}}$ & 1 & .007 \\
\hline Continuity Correction $^{\circ}$ & 5.879 & 1 & .015 \\
\hline Likelihood Ratio & 6.814 & 1 & .009 \\
\hline \multicolumn{4}{|l|}{ Fisher's Exact Test } \\
\hline Linear-by-Linear Association & 7.202 & 1 & .007 \\
\hline $\mathrm{N}$ of Valid Cases & 107 & & \\
\hline
\end{tabular}

Question 4: How much are consumers willing to spend on a purchase of a certified pre-owned vehicle?

Univariate Analysis in Table 11 shows that $30.8 \%$ of

respondents are willing to spend over $\$ 20,000$ and $26.2 \%$ are willing to spend $\$ 10,000-\$ 15,000$ on a pre-owned vehicle.

Table 11: Amount Customer is Willing to Spend on a Pre-owned Vehicle

\begin{tabular}{|l|c|c|}
\hline \multicolumn{1}{|c|}{ Variable } & \# of Respondents & $\%$ of Respondents \\
\hline Below $\$ 10,000$ & 6 & $5.6 \%$ \\
\hline$\$ 10,000-\$ 15,000$ & 28 & $26.2 \%$ \\
\hline$\$ 15,001-\$ 20,000$ & 33 & $19.6 \%$ \\
\hline Over \$20,000 & 18 & $30.8 \%$ \\
\hline $\begin{array}{l}\text { I would not purchase a pre-owned } \\
\text { vehicle }\end{array}$ & 106 & $16.8 \%$ \\
\hline Total & 21 & $99.1 \%$ \\
\hline
\end{tabular}

Using ANOVA to look at Customer's Willingness to Purchase Pre-Owned Vehicle and Willingness to Pay for a Pre-Owned 
Vehicle (Table 12 and 13), we fail to reject the Ho, as consumers who are willing to purchase a used vehicle are motivated to spend $\$ 15,000-\$ 20,000$ on the purchase of a pre-owned vehicle.

Table 12: ANOVA Between Customer's Willingness to Purchase Pre-Owned Vehicle and Willingness to Pay for a Pre-Owned Vehicle

Table 13: ANOVA Customer's Willingness to Purchase Pre-Owned Vehicle and

\begin{tabular}{|c|c|c|c|c|c|c|c|c|}
\hline \multicolumn{9}{|c|}{ Descriptives } \\
\hline & \multirow[b]{2}{*}{$\mathrm{N}$} & \multirow[b]{2}{*}{ Mean } & \multirow[b]{2}{*}{$\begin{array}{c}\text { Std. } \\
\text { Deviation }\end{array}$} & \multirow[b]{2}{*}{$\begin{array}{l}\text { Std. } \\
\text { Error }\end{array}$} & \multicolumn{2}{|c|}{$\begin{array}{l}95 \% \text { Confidence } \\
\text { Interval for Mean }\end{array}$} & \multirow[b]{2}{*}{ Minimum } & \multirow[b]{2}{*}{ Maximum } \\
\hline & & & & & \begin{tabular}{l|} 
Lower \\
Bound
\end{tabular} & $\begin{array}{l}\text { Upper } \\
\text { Bound }\end{array}$ & & \\
\hline$\$ 15,000$ or less & 32 & 3.0000 & 1.10716 & .19572 & 2.6008 & 3.3992 & 1.00 & 5.00 \\
\hline$\$ 15,001-\$ 20,000$ & 20 & 2.2000 & 1.00525 & .22478 & 1.7295 & 2.6705 & 1.00 & 5.00 \\
\hline Over $\$ 20,000$ & 30 & 2.3333 & 1.37297 & .25067 & 1.8207 & 2.8460 & 1.00 & 5.00 \\
\hline $\begin{array}{l}\text { would not purchase } \\
\text { a pre-owned vehicle }\end{array}$ & 18 & 2.5556 & 1.19913 & .28264 & 1.9592 & 3.1519 & 1.00 & 5.00 \\
\hline Total & 100 & 2.5600 & 1.21705 & .12171 & 2.3185 & 2.8015 & 1.00 & 5.00 \\
\hline
\end{tabular}

Willingness to Pay for a Pre-Owned Vehicle

\begin{tabular}{|l|r|r|r|r|r|}
\hline & \multicolumn{1}{|c|}{$\begin{array}{c}\text { Sum of } \\
\text { Squares }\end{array}$} & \multicolumn{1}{c|}{$\mathrm{df}$} & \multicolumn{1}{c|}{$\begin{array}{c}\text { Sean } \\
\text { Square }\end{array}$} & \multicolumn{1}{c|}{$\mathrm{F}$} & Sig. \\
\hline Between & 10.329 & 3 & 3.443 & 2.425 & .070 \\
Groups & 136.311 & 96 & 1.420 & & \\
Within Groups & 146.640 & 99 & & & \\
Total & & & \\
\hline
\end{tabular}

Question 5: How much would consumers pay overall for a Certified Pre-Owned which includes their preferred features? Other preferred features that respondents desired included 24-hour tech support, a better warranty, and complimentary maintenance (Table 14). Univariate analysis in Table 15 reveals that $36.4 \%$ are willing to pay $\$ 1000$ - $\$ 1999$ more on a CPO over a non-CPO Vehicle, and $25.2 \%$ were willing to spend under $\$ 999$. 


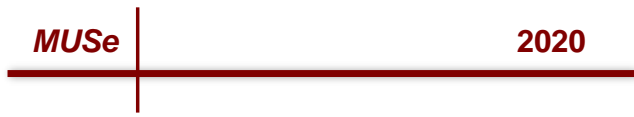

Table 14: Other Options Customers Would Like to See Included in a Certified Pre-owned Vehicle

\begin{tabular}{|l|c|c|}
\hline \multicolumn{1}{|c|}{ Variable } & $\begin{array}{c}\# \text { of } \\
\text { Respondents }\end{array}$ & \% of Respondents \\
\hline $\begin{array}{l}\text { 1-year maintenance free and oil change for } \\
\text { life }\end{array}$ & 1 & $.9 \%$ \\
\hline 24-hour Tech support & 1 & $.9 \%$ \\
\hline A better warranty & 1 & $.9 \%$ \\
\hline Coffee and biscuits & 1 & $.9 \%$ \\
\hline Complimentary maintenance & 1 & $.9 \%$ \\
\hline $\begin{array}{l}\text { Discounted prices for scratch/rock chip } \\
\text { repair }\end{array}$ & 1 & $.9 \%$ \\
\hline $\begin{array}{l}\text { Last tire changes especially for winter tires. } \\
\text { Many people do not include that when } \\
\text { selling pre-owned vehicles. }\end{array}$ & 1 & $.9 \%$ \\
\hline $\begin{array}{l}\text { Leather seats } \\
\text { mats, anything to make the old ride look }\end{array}$ & 1 & $.9 \%$ \\
\hline New tires, repairs up to date & 1 & $.9 \%$ \\
\hline Sun roof & 1 & $.9 \%$ \\
\hline Tires or other features like them. & 1 & \\
\hline Warranty from dealership & 1 & $.9 \%$ \\
\hline
\end{tabular}

Table 15: Amount Customer Willing to Spend For a Certified Pre-Owned Over a Non-Certified Pre-Owned Vehicle 
Kasawski, Singh, Bhullar, Sidhu, Bath

\begin{tabular}{|l|c|c|}
\hline \multicolumn{1}{|c|}{ Variable } & \# of Respondents & \% of Respondents \\
\hline Under \$999 & 27 & $25.2 \%$ \\
\hline$\$ 1000-\$ 1999$ & 39 & $36.4 \%$ \\
\hline$\$ 2000-\$ 2999$ & 14 & $13.1 \%$ \\
\hline$\$ 3000$ - \$3999 & 3 & $2.8 \%$ \\
\hline$\$ 4000+$ & 8 & $7.5 \%$ \\
\hline $\begin{array}{l}\text { I would not purchase a pre- } \\
\text { owned vehicle }\end{array}$ & 3 & $2.8 \%$ \\
\hline $\begin{array}{l}\text { I would not spend more on a } \\
\text { certified pre-owned vehicle }\end{array}$ & 13 & $12.1 \%$ \\
\hline Total & 107 & $100.0 \%$ \\
\hline
\end{tabular}

After we conducted and analyzed the multiple response cross tabulation (Table 16), we determined that consumers were willing to pay more for their preferred features.

Complimentary oil \& filter service and made within last 7 years will increase percentage of people willing to pay $\$ 15000$ or less. CarProof verification will do so for those who are willing to pay $\$ 15,001-\$ 20,000$. Enrollment with dealership exclusives Five/Ten (5-10) day grace period and Full tank of gas will increase percentage of people willing to pay over $\$ 20,000$. Therefore, we fail to reject the Ho. 
Table 16: Multiple Response Cross Tabulation between Customer's Willingness to Purchase Used Vehicle and CPO feature Preferences

\begin{tabular}{|c|c|c|c|c|c|c|}
\hline \multicolumn{6}{|c|}{\begin{tabular}{c|c}
${ }^{2}$ & $\begin{array}{c}\text { Willingness to Pay Overall } \\
\text { Collapsed }\end{array}$ \\
\cline { 2 - 3 }
\end{tabular}} & \multirow[b]{2}{*}{ Total } \\
\hline & & & $\begin{array}{l}515,000 \\
\text { or less }\end{array}$ & $\begin{array}{l}\$ 15,001 * \\
520,000\end{array}$ & $\begin{array}{c}\text { Over } \\
\$ 20,000\end{array}$ & \\
\hline \multirow{20}{*}{$\begin{array}{l}\text { CPO } \\
\text { Preferred } \\
\text { Options }\end{array}$} & \multirow{2}{*}{$\begin{array}{l}4 \text { montha/40K km Powertran } \\
\text { and Rosdside Assistance } \\
\text { coverage }\end{array}$} & Count & 12 & 10 & 16 & 38 \\
\hline & & $\begin{array}{l}\text { W within CPO } \\
\text { Preferred } \\
\text { Options }\end{array}$ & $31.6 \%$ & $25.3 \%$ & $42.1 \%$ & \\
\hline & \multirow{2}{*}{$\begin{array}{l}\text { CarProct verif cation" includes } \\
\text { accident report, recal, lens, ebc. }\end{array}$} & Count & 21 & 19 & 24 & 64 \\
\hline & & $\begin{array}{l}\text { W within CPO } \\
\text { Preferred } \\
\text { Options }\end{array}$ & $328 \%$ & $29.7 \%$ & $37.5 \%$ & \\
\hline & \multirow[t]{2}{*}{ Complimentary ol s stler service } & Count & $1 \mathrm{~B}$ & 12 & 12 & 42 \\
\hline & & $\begin{array}{l}\text { W within CPO } \\
\text { Preferred } \\
\text { Options }\end{array}$ & $429 \%$ & $28.6 \%$ & 28.655 & \\
\hline & \multirow{2}{*}{$\begin{array}{l}\text { Enrolment with dealership } \\
\text { exclusives, including priwate } \\
\text { events, tree rentals, and VIP } \\
\text { services }\end{array}$} & Count & 7 & 4 & 11 & 22 \\
\hline & & $\begin{array}{l}\text { W within CPO } \\
\text { Preferned } \\
\text { Options }\end{array}$ & $31.8 \%$ & $18.2 \%$ & $50.0 \% 5$ & \\
\hline & \multirow{2}{*}{$\begin{array}{l}\text { Five/Ten (5-10) dayj1500km } \\
\text { (whichever comes firs:) grace } \\
\text { period for swap or trade }\end{array}$} & Count & 13 & $B$ & 19 & 40 \\
\hline & & $\begin{array}{l}\text { W within CPO } \\
\text { Preferned } \\
\text { Options }\end{array}$ & $32.5 \%$ & $20.0 \%$ & $47.5 \%$ & \\
\hline & \multirow[t]{2}{*}{ Full tank of gas } & Count & है & 2 & 9 & 17 \\
\hline & & $\begin{array}{l}\text { X within CPO } \\
\text { Preferred } \\
\text { Options }\end{array}$ & $35.3 \%$ & $11.8 \%$ & $52.5 \%$ & \\
\hline & \multirow[t]{2}{*}{ Made w thin the last 7 years } & Count & 25 & 16 & 20 & 61 \\
\hline & & $\begin{array}{l}\text { W within CPO } \\
\text { Preferned } \\
\text { Options }\end{array}$ & $41.0 \%$ & $25.2 \%$ & $32.8 \%$ & \\
\hline & \multirow[t]{2}{*}{ One yoar end to-end warranty } & Count & 18 & 17 & 24 & 58 \\
\hline & & $\begin{array}{l}\text { \% within CPO } \\
\text { Prefemed } \\
\text { Options }\end{array}$ & $30.5 \%$ & $2 B .8 \%$ & $40.7 \%$ & \\
\hline & \multirow[t]{2}{*}{ Less than $110 \mathrm{~K}$ klometers } & Count & 0 & 1 & 0 & 1 \\
\hline & & $\begin{array}{l}\text { W within CPO } \\
\text { Preferred } \\
\text { Options }\end{array}$ & $0.0 \%$ & $100.0 \%$ & $0.0 \%$ & \\
\hline & \multirow[t]{2}{*}{ Less than BOK klometers } & Count & $2 B$ & 19 & 24 & 71 \\
\hline & & $\begin{array}{l}\text { \% within CPO } \\
\text { Preferred } \\
\text { Options }\end{array}$ & $39.4 \%$ & $25.8 \%$ & $33.8 \%$ & \\
\hline \multirow{2}{*}{\multicolumn{2}{|c|}{ Total }} & Count & 148 & 108 & 159 & 415 \\
\hline & & & $35.7 \%$ & $26 \%$ & $38.3 \%$ & \\
\hline
\end{tabular}


Question 6: Will gender affect their preference paying more for used over certified pre-owned vehicles if the CPO costs more? Cross-tabulation results in Table 17 and chi-square test in Table 18 indicate there is a positive correlation between total amount of consumers and willingness to pay $\$ 1000$ - \$1999 for a CPO vehicle over a non-CPO (42.9\%). Additionally, $64.1 \%$ of males chose, while only $33.3 \%$ of females chose $\$ 1000$ - $\$ 1999$, preferring to spend $\$ 999$ and Under (37.0\%). Therefore, we fail to reject the Ho.

Table 17: Cross Tabulation between Willingness to Pay more for CPO over non-CPO Vehicles and Gender

Willingness to Pay More for CPO and Gender

\begin{tabular}{|c|c|c|c|c|c|c|}
\hline & \multicolumn{3}{|c|}{ Gender } & \multirow[b]{2}{*}{ Total } \\
\hline & & & Female & Male & $\begin{array}{l}\text { Prefer not } \\
\text { to answer }\end{array}$ & \\
\hline \multirow{9}{*}{$\begin{array}{l}\text { Willingness } \\
\text { to Pay More } \\
\text { for CPO }\end{array}$} & Under & Count & 10 & 17 & 0 & 27 \\
\hline & $\$ 999$ & $\%$ within Pay More for CPO & $37.0 \%$ & $63.0 \%$ & $0.0 \%$ & $100.0 \%$ \\
\hline & & $\%$ within Gender & $28.6 \%$ & $32.7 \%$ & $0.0 \%$ & $29.7 \%$ \\
\hline & $\$ 1000$ & Count & 13 & 25 & 1 & 39 \\
\hline & - & $\%$ within Pay More for CPO & $33.3 \%$ & $64.1 \%$ & $2.6 \%$ & $100.0 \%$ \\
\hline & $\$ 1999$ & $\%$ within Gender & $37.1 \%$ & $48.1 \%$ & $25.0 \%$ & $42.9 \%$ \\
\hline & $\$ 2000$ & Count & 12 & 10 & 3 & 25 \\
\hline & + & $\%$ within Pay More for CPO & $48.0 \%$ & $40.0 \%$ & $12.0 \%$ & $100.0 \%$ \\
\hline & & $\%$ within Gender & $34.3 \%$ & $19.2 \%$ & $75.0 \%$ & $27.5 \%$ \\
\hline Total & & Count & 35 & 52 & 4 & 91 \\
\hline
\end{tabular}




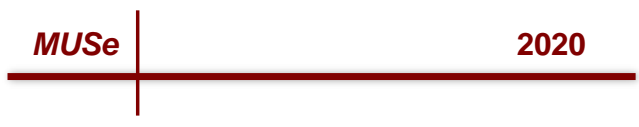

Table 18: Chi- Square Test

\begin{tabular}{|c|c|c|c|}
\hline \multicolumn{4}{|c|}{ Chi-Square Test } \\
\hline & Value & df & $\begin{array}{c}\text { Asymptotic } \\
\text { Significance } \\
\text { (2-sided) }\end{array}$ \\
\hline Pearson Chi-Square & $7.422^{2}$ & 4 & .115 \\
\hline Likelihood Ratio & 7.700 & 4 & .103 \\
\hline $\begin{array}{l}\text { Linear-by-Linear } \\
\text { Association }\end{array}$ & .006 & 1 & .937 \\
\hline $\mathrm{N}$ of Valid Cases & 91 & & \\
\hline
\end{tabular}

a. 3 cells $(33.3 \%)$ have expected count less than 5 . The minimum expected count is 1.10 .

Question 7: What would consumers like to see when advertising certified pre-owned vehicles to their customers? Table 19 univariate analysis shows that $27.1 \%$ of respondents found CarProof verification certified pre-owned vehicle advertising the most appealing, followed by Lexus of Edmonton certified pre-owned vehicle (26.2\%), and 210-point check certified pre-owned vehicle (19.6\%). The preferred method for communications was text at $34.6 \%$, followed closely by email at $32.7 \%$ (Table 20 ).

Table 19: Advertisements Most Appealing to Customers

\begin{tabular}{|l|c|c|}
\hline \multicolumn{1}{|c|}{ Variable } & \# of Respondents & \% of Respondents \\
\hline $\begin{array}{l}\text { 131-point check certified pre-owned } \\
\text { vehicle }\end{array}$ & 6 & $5.6 \%$ \\
\hline $\begin{array}{l}\text { 210-point check certified pre-owned } \\
\text { vehicle }\end{array}$ & 21 & $19.6 \%$ \\
\hline $\begin{array}{l}\text { CarProof verification certified pre- } \\
\text { owned vehicle }\end{array}$ & 29 & $27.1 \%$ \\
\hline
\end{tabular}


Kasawski, Singh, Bhullar, Sidhu, Bath

\begin{tabular}{|l|c|c|}
\hline \multicolumn{1}{|c|}{ Variable } & \# of Respondents & \% of Respondents \\
\hline $\begin{array}{l}\text { Lexus of Edmonton certified pre- } \\
\text { owned vehicle }\end{array}$ & 28 & $26.2 \%$ \\
\hline None of these appeal to me & 21 & $19.6 \%$ \\
\hline Total & 105 & $98.1 \%$ \\
\hline
\end{tabular}

Table 20: Customers Preferred Method of Contact for Communication

\begin{tabular}{|l|c|c|}
\hline \multicolumn{1}{|c|}{ Variable } & \# of Respondents & $\%$ of Respondents \\
\hline Email & 35 & $32.7 \%$ \\
\hline Phone Call & 25 & $23.4 \%$ \\
\hline Text & 37 & $34.6 \%$ \\
\hline Total & 97 & $90.7 \%$ \\
\hline
\end{tabular}

Based on cross-tabulation and chi-square test (Table 21 and $22)$, results show that consumers care most about advertising CPO vehicles with CarProof verification (27.6\%), followed closely by a CPO vehicle featured with Lexus of Edmonton (26.7\%). Consumers with certification knowledge are positively correlated with CPO vehicles being sold at Lexus of Edmonton (31.9\%) initially seen in advertisements. Respondents unknowledgeable in certification tied in preference with certification points (12.1\% each). We can reject our Ho. Advertisements showing 210-point check, CarProof and Lexus of Edmonton would work well together. 
Table 21: Cross Tabulation Between Consumers Certification Knowledge and Desired Advertising

\begin{tabular}{|c|c|c|c|c|c|c|c|c|}
\hline & & & \multicolumn{5}{|c|}{ Desired Advertising } & \multirow[b]{2}{*}{ Total } \\
\hline & & & $\begin{array}{l}131 \text { point } \\
\text { check } \\
\text { certified } \\
\text { pre-owned } \\
\text { vehicle } \\
\end{array}$ & $\begin{array}{l}210 \text { point } \\
\text { check } \\
\text { certified } \\
\text { pre-owned } \\
\text { vehicle } \\
\end{array}$ & $\begin{array}{l}\text { CarProof } \\
\text { verification } \\
\text { certified } \\
\text { pre-owned } \\
\text { vehicle }\end{array}$ & $\begin{array}{l}\text { Lexus of } \\
\text { Edmonton } \\
\text { certified } \\
\text { pre-owned } \\
\text { vehicle } \\
\end{array}$ & $\begin{array}{c}\text { None of } \\
\text { these appeal } \\
\text { to me }\end{array}$ & \\
\hline \multirow{6}{*}{$\begin{array}{l}\text { Certification } \\
\text { Knowledge }\end{array}$} & \multirow{3}{*}{ No } & \multirow{3}{*}{$\begin{array}{l}\text { Count } \\
\text { Expected Count } \\
\% \text { within } \\
\text { Certification } \\
\text { Knowledge }\end{array}$} & 4 & 4 & 8 & 5 & 12 & 33 \\
\hline & & & 1.9 & 6.6 & 9.1 & 8.8 & 6.6 & 33.0 \\
\hline & & & $12.1 \%$ & $12.1 \%$ & $24.2 \%$ & $15.2 \%$ & $36.4 \%$ & $100.0 \%$ \\
\hline & \multirow[t]{3}{*}{ Yes } & Count & 2 & 17 & 21 & 23 & 9 & 72 \\
\hline & & Expected Count & 4.1 & 14.4 & 19.9 & 19.2 & 14.4 & 72.0 \\
\hline & & $\begin{array}{l}\text { \% within } \\
\text { Certification } \\
\text { Knowledge }\end{array}$ & $2.8 \%$ & $23.6 \%$ & $29.2 \%$ & $31.9 \%$ & $12.5 \%$ & $100.0 \%$ \\
\hline \multirow{3}{*}{\multicolumn{2}{|c|}{ Total }} & Count & 6 & 21 & 29 & 28 & 21 & 105 \\
\hline & & Expected Count & 6.0 & 21.0 & 29.0 & 28.0 & 21.0 & 105.0 \\
\hline & & $\begin{array}{l}\% \text { within } \\
\text { Certification } \\
\text { Knowledge }\end{array}$ & $5.7 \%$ & $20.0 \%$ & $27.6 \%$ & $26.7 \%$ & $20.0 \%$ & $100.0 \%$ \\
\hline
\end{tabular}

Table 22: Chi-Square Test

\section{Chi-Square Tests}

\begin{tabular}{|c|c|c|c|}
\hline & Value & $\mathrm{df}$ & $\begin{array}{c}\text { Asymptotic } \\
\text { Significance (2- } \\
\text { sided) }\end{array}$ \\
\hline Pearson Chi-Square & $13.986^{\mathrm{a}}$ & 4 & .007 \\
\hline Likelihood Ratio & 13.513 & 4 & .009 \\
\hline Linear-by-Linear Association & .921 & 1 & .337 \\
\hline N of Valid Cases & 105 & & \\
\hline
\end{tabular}


Kasawski, Singh, Bhullar, Sidhu, Bath

Question 8: Do consumers who trust Lexus of Edmonton value certified and non-certified pre-owned vehicles equally?

Based on Table 23, univariate analysis shows that $55.1 \%$ of respondents had never been to Lexus of Edmonton dealership. $35.5 \%$ of the $44.9 \%$ of those who had been to the dealership were Very Satisfied or Somewhat Satisfied with the dealership's customer service, as seen in Table 24.

Table 23: Amount of Customers Who Have Been to the Lexus of Edmonton Dealership

\begin{tabular}{|l|c|c|}
\hline \multicolumn{1}{|c|}{ Variable } & \# of Respondents & \% of Respondents \\
\hline No & 59 & $55.1 \%$ \\
\hline Yes & 48 & $44.9 \%$ \\
\hline Total & 107 & $100.0 \%$ \\
\hline
\end{tabular}

Table 24: Customers Level of Satisfaction with Lexus of Edmonton's Customer Service

\begin{tabular}{|l|c|c|}
\hline \multicolumn{1}{|c|}{ Variable } & \# of Respondents & \% of Respondents \\
\hline Very Satisfied & 21 & $19.6 \%$ \\
\hline Somewhat Satisfied & 17 & $15.9 \%$ \\
\hline Neither Satisfied or Dissatisfied & 7 & $6.5 \%$ \\
\hline Somewhat Dissatisfied & 1 & $.9 \%$ \\
\hline Very Dissatisfied & 2 & $1.9 \%$ \\
\hline Total & 48 & $44.9 \%$ \\
\hline
\end{tabular}

An ANOVA analysis in Table 25 and 26 was used for Consumers' Trust with Lexus of Edmonton and Willingness to Purchase CPO over non-CPO Vehicles. A significant difference between the yes and no responses regarding the trust of Lexus 


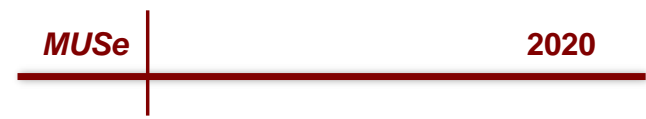

of Edmonton's pre-owned dealership is not evident. We fail to reject the Ho. Those who have knowledge of certified preowned tend to trust dealerships more than those who do not have knowledge of certified pre-owned.

Table 25: ANOVA for Consumers' Trust with Lexus of Edmonton and Willingness to Purchase CPO over non-CPO Vehicles

Table 26: ANOVA

Descriptives

Scale $1-5$ with 1 being 'no trust' and 5 being 'complete trust'

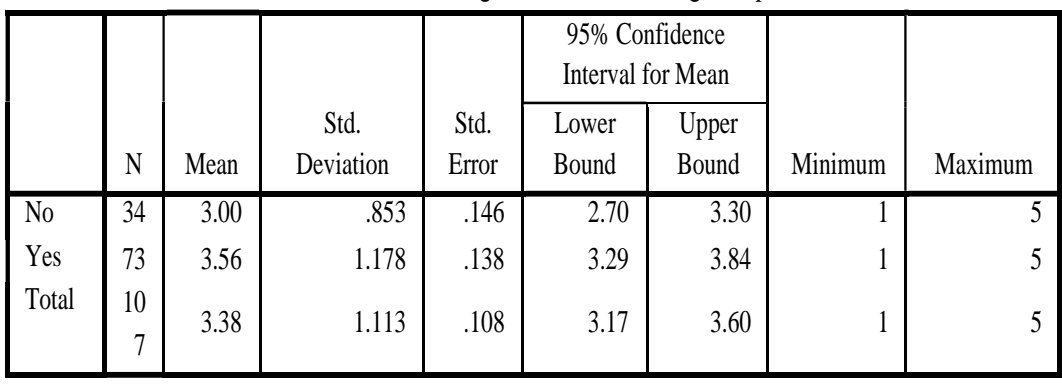

Scale 1-5 with 1 being 'no trust' and 5 being 'complete trust'

\begin{tabular}{|l|r|r|r|r|r|}
\hline & Sum of Squares & df & Mean Square & \multicolumn{1}{c|}{$\mathrm{F}$} & Sig. \\
\hline Between Groups & 7.317 & 1 & 7.317 & 6.197 & .014 \\
Within Groups & 123.973 & 105 & 1.181 & & \\
Total & 131.290 & 106 & & & \\
\hline
\end{tabular}

Question 9: Does trust in Lexus of Edmonton increase consumers' willingness to pay more for a Certified Pre-owned Vehicle?

Based on the ANOVA results for Consumers Trust with Lexus of Edmonton and Willingness to Pay more for Certified PreOwned Vehicle (Table 27 and 28), there is no relationship between incremental price points and trust. Therefore, we reject the Ho. 
Kasawski, Singh, Bhullar, Sidhu, Bath

Table 27: ANOVA for Consumers Trust with Lexus of Edmonton and Willingness to Pay more for Certified Pre-Owned Vehicle

Table 28: ANOVA

\section{Descriptives}

Scale $1-5$ with 1 being 'no trust' and 5 being 'complete trust'

\begin{tabular}{|c|c|c|c|c|c|c|c|c|}
\hline & \multirow[b]{2}{*}{$\mathrm{N}$} & \multirow[b]{2}{*}{ Mean } & \multirow{2}{*}{$\begin{array}{c}\text { Std. } \\
\text { Deviation }\end{array}$} & \multirow{2}{*}{$\begin{array}{l}\text { Std. } \\
\text { Error }\end{array}$} & \multicolumn{2}{|c|}{$\begin{array}{l}95 \% \text { Confidence Interval for } \\
\text { Mean }\end{array}$} & \multirow[b]{2}{*}{ Minimum } & \multirow[b]{2}{*}{ Maximum } \\
\hline & & & & & Lower Bound & Upper Bound & & \\
\hline Under $\$ 999$ & 27 & 3.44 & .974 & .187 & 3.06 & 3.83 & 1 & 5 \\
\hline $\begin{array}{l}\$ 1000- \\
\$ 1999\end{array}$ & 39 & 3.33 & .982 & .157 & 3.01 & 3.65 & 1 & \\
\hline$\$ 2000+$ & 25 & 3.68 & .945 & .189 & 3.29 & 4.07 & 2 & 5 \\
\hline Total & 91 & 3.46 & .970 & .102 & 3.26 & 3.66 & 1 & 5 \\
\hline
\end{tabular}

Scale 1-5 with 1 being 'no trust' and 5 being 'complete trust'

\begin{tabular}{|l|r|r|r|r|r|}
\hline & Sum of Squares & \multicolumn{1}{|c|}{ df } & Mean Square & F & \multicolumn{1}{c|}{ Sig. } \\
\hline Between Groups & 1.842 & 2 & .921 & .979 & .380 \\
Within Groups & 82.773 & 88 & .941 & & \\
Total & 84.615 & 90 & & & \\
\hline
\end{tabular}

Question 10: Do consumers trust Lexus of Edmonton in selling them a certified pre-owned vehicle?

Univariate analysis shows that on a scale of 1 to 5 , with 1 being no trust and 5 being complete trust, $47.7 \%$ of respondents chose 4 or 5 , trusting Lexus of Edmonton in selling them a used vehicle (Table 29).

Table 29: Customer's Level of Trust in Lexus of Edmonton in Selling Them a Used Vehicle

\begin{tabular}{|l|l|l|}
\hline Variable & \# of Respondents & $\begin{array}{c}\% \text { of } \\
\text { Respondents }\end{array}$ \\
\hline
\end{tabular}




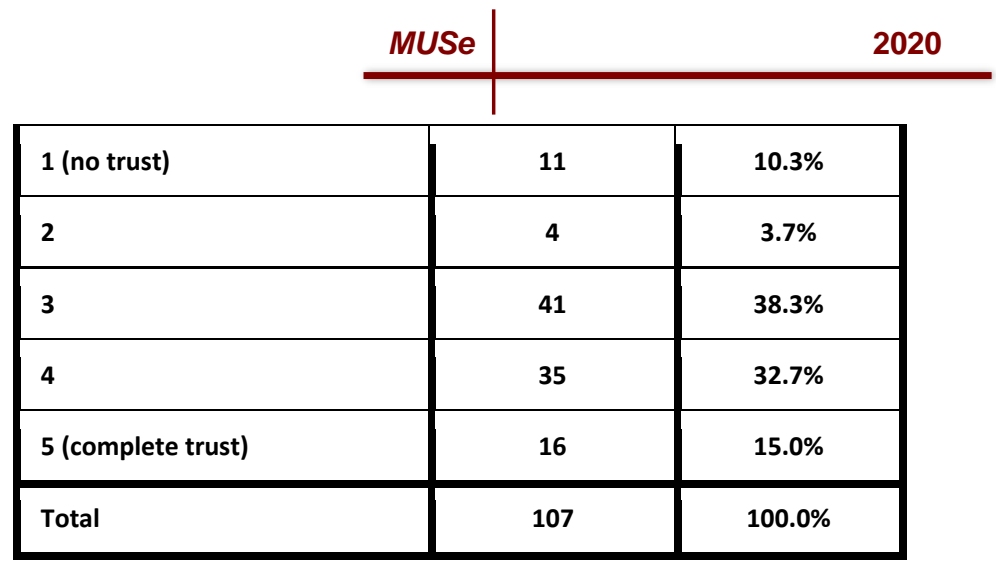

There is a significant relationship between satisfaction with Lexus and Trust, based on the Pearson correlation in Table 30. The coefficient of correlation is negative because trust has an inverse scale (1-no trust to 5-complete trust) compared to satisfaction with Lexus (1-very satisfied to 5 -very dissatisfied). This is consistent with our hypothesis that the majority of consumers will purchase a certified pre-owned vehicle from a dealership they trust. We reject the Ho. 
Table 30: Pearson correlation for Consumers' Trust and Level of Satisfaction with Lexus of Edmonton

\begin{tabular}{|c|c|c|c|}
\hline & & $\begin{array}{l}\text { Scale } 1-5 \\
\text { with } 1 \\
\text { being 'no } \\
\text { trust' and } 5 \\
\text { being } \\
\text { 'complete } \\
\text { trust' }\end{array}$ & $\begin{array}{c}\text { Level } \\
\text { Satisfaction } \\
\text { with Lexus } \\
\text { of Edmonton }\end{array}$ \\
\hline $\begin{array}{l}\text { Scale } 1-5 \text { with } 1 \\
\text { being 'no trust' and } 5 \\
\text { being 'complete } \\
\text { trust' }\end{array}$ & $\begin{array}{l}\text { Pearson } \\
\text { Correlation } \\
\text { Sig. (2-tailed) } \\
\text { N }\end{array}$ & 107 & $\begin{array}{r}-.636 \\
.000 \\
48\end{array}$ \\
\hline Q12_recoded & $\begin{array}{l}\text { Pearson } \\
\text { Correlation } \\
\text { Sig. (2-tailed) } \\
\text { N }\end{array}$ & $\begin{array}{r}-636^{4} \\
.000 \\
48\end{array}$ & 1 \\
\hline
\end{tabular}

**. Correlation is significant at the 0.01 level (2-tailed).

\section{Conclusions and Recommendations}

The objective of the current research problem was to identify if Certified Pre-Owned vehicles added value to Lexus of Edmonton. We conducted two stages of research: secondary and primary. In the first stage, secondary research, we reviewed the literature. The second stage included interviewing Lexus of Edmonton management, in-depth interviews, and surveys. With the use of SPSS, we were able to analyze the data in an effective manner. Analyzing these results, we were able to break down the information into meaningful data that will assist in addressing Lexus of Edmonton's CPO vehicle concern. After analyzing our findings, we recommend the following when dealing with CPO vehicle sales for the 25 and under demographic:

- Lexus of Edmonton should educate used-vehicle consumers on their certified pre-owned program and its benefits, as well as what is included. 


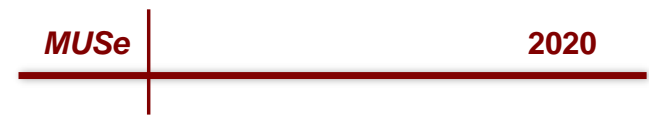

- Lexus should price accordingly to their target market, with a focus on pre-owned vehicles in the range of $\$ 15,000$ - \$20,000.

- We suggest that LoE should price their CPO vehicles no more than $\$ 1999$ over non-CPO vehicles.

- Lexus should consider offering less than 80K, CarProof verification, one-year end-to-end warranty, and made within the last 7 years as the top four options for CPO vehicles. When choosing additional value benefits, we suggest LoE look at what each price market values the most. Complimentary oil \& filter service and made within last 7 years are highly marketable for consumers willing to pay $\$ 15000$ or less. CarProof verification is highly marketable for consumers willing to pay $\$ 15,001-\$ 20,000$. Enrollment with dealership exclusives Five/Ten (5-10) day grace period and Full tank of gas are highly marketable for consumers willing to pay over $\$ 20,000$.

- Lexus of Edmonton should continue raising its brand awareness by advertising its CPO vehicles with CarProof Verification and the trusted Lexus of Edmonton symbol, for those willing to pay $\$ 15,000$ $\$ 20,000$.

- Lexus of Edmonton should continue further marketing research on what platforms their targeted markets interact with. Further marketing research needs to be conducted on how to maximize building consumer trust.

Based on our findings, we believe that $\mathrm{CPO}$ vehicles can add value to Lexus of Edmonton if they instill the recommended value-added properties included above. By building trust and listening to the needs of the market, Lexus of Edmonton will continue to be an exemplary luxury dealership. 
VI. Limitations

As with any research project, we encountered certain research limitations while conducting exploratory research. The following limitations have been taken into consideration:

1. Accuracy - Anonymous online surveys cannot measure rate of accuracy. This includes age, gender, occupation or location. We cannot guarantee authenticity of responses collected by our questionnaire.

2. Sample size - In further research, with a less constraining time limit, we could have conducted more surveys, which would have led to a larger sample size; therefore, our data would have had a higher degree of variability.

Although accuracy and sample size presented our research with limitations, they are minimal aspects that could have been monitored. After conducting exploratory and secondary research, we are satisfied with the results obtained. 


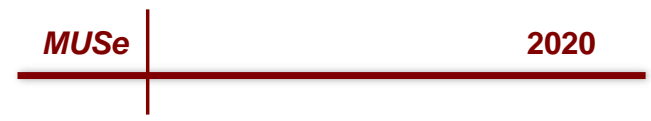

\section{References}

Ažman, S., \& Gomišček, B. (2014). Functional form of

connections between perceived service quality, customer satisfaction and customer loyalty in the automotive servicing industry. Total Quality Management \& Business Excellence, 26(7-8), 888-904. https://doi.org/10.1080/14783363.2014.909172t

Busse, M., Knittel, C., \& Zettelmeyer, F. (2013). Are consumers myopic? Evidence from new and used car purchases. American Economic Review, 103(1), 220-256. https://doi.org/10.1257/aer.103.1.220

Barkholz, D. (2015). Used-car powerhouse. Automotive News, 89(6695), S016. http://www.autonews.com

Chappell, L. (2015). Dealer: Color-coding cars makes shopping easier. Automotive News, 89(6688), 0014.

http://www.autonews.com.

Confente, I., \& Russo, I. (2015). After-sales service as a driver

for word-of-mouth and customer satisfaction: Insights from the automotive industry. International Journal of Management Cases, 17(4), 59-72. www.ijmc.org

Cristian, D. D. \& Nicoleta, A. I., (2011). Study on retail brand awareness in retail. Annals of the University of Oradea, Economic Science Series, 20(2). 742-748. http://anale.steconomiceuoradea.ro

Fan, J. X., \& Burton, J. R. (2005). Vehicle acquisitions: Leasing or financing?. Journal Of Consumer Affairs, 39(2), 237-253. http://www.ebscohost.com

Hsiao, Y. H., Hsu, Y., \& Fang, W., (2014). Is brand awareness a marketing placebo?. International Journal of Business \& Information. 9(1), 29-60. http://www.knowledgetaiwan.org 
Jones, L. (1997). Used car dealers adopt certification programs, expect sales jump. Enterprise/Salt Lake City, 27(9),1. https://www.ebscohost.com

Lacetera, N., Pope, D., \& Sydnor, J. (2012). Heuristic thinking and limited attention in the car market. American Economic Review, 102(5), 2206 - 2236. https://doi.org/ 10.1257/aer.102.5.2206

Lewis, E. (2015, December 9). Nearly 9 out of 10 used car shoppers willing to consider certified pre-owned vehicles, says Edmunds.com. http://www.edmunds.com

Mcdonald, M. (2012). Internet reshapes how dealers sell used cars. WardsAuto Dealer Business, 46(9), 16-18. http://wardsauto.com

Mattera, M., Baena, V., \& Cerviño, J., (2012). Analyzing social responsibility as a driver of firm's brand awareness. Procedia- social and behavioural sciences, 58(1), 1121-1130.

Nemtuda, D. (2013). Certified Pre-Owned: The ripple effect in the automotive industry. https://www.experian.com

Peterson, J. \& Schneider, H. (2014). Adverse selection in the used-car market: Evidence from purchase and repair patterns in the consumer expenditure survey. RAND Journal of Economics, 45 (1), 140154. https://doi.org/10.1111/1756-2171.12045

Peters, E. (2002). Certified, pre-owned cars: 'Used' doesn't mean 'abused'. Consumers' Research Magazine. 85(11). 32-33. http://consumersresearch.org

Shipp, J. (2013, December 5). New autotrader.com study shows key changes in shoppers' familiarity, opinion and consideration of cpo vehicles. http://press.autotrader.com

Sawyers, A. (2010). New life from used cars. Automotive News, 84(6408), 15-29. http://www.autonews.com 
Sawyers, A. \& Treece, J.B. (2016). Lexus is flexing its CPO muscles. Automotive News, 90(6706), 8. http://www.autonews.com

Sultan, A. (2010). Lemons and certified pre-owned cars in the used car market. Applied Economics Letters, 17(1). 45-50. https://doi.org/10.1080/13504850701719678.

Strautmanis, J. \& Hermanis, J. (2013). Young consumers:

Financial potential and preferred advisors. Journal Of Business Management, (7), 141-151. http://www.ebscohost.com

Zarantonello, L., Schmitt, B. H., \& Jedidi, K. (2014). How to advertise and build brandknowledge globally? Journal of Advertising Research JAR, 54(4), 420434. https://doi.org/10.2501/JAR-54-4-420-434 


\section{Appendix Questionnaire}

This questionnaire is part of a research project which gathers relevant data and assists Lexus of Edmonton towards improving their current business model within the certified pre-owned vehicle unit. Reading the description and completing the questionnaire indicates that you are giving your informed consent to participate in the study. The consent, however, does not constitute a waiver of legal rights in the event of research-related harm.

Project Title: Do certified pre-owned vehicles add value to Lexus of Edmonton, and if so, is it an investment worth making?

Researchers: 5 Authors, MacEwan University

\section{Purpose of the Research:}

The focus of this study is to understand the value of certified pre-owned vehicles for Lexus, with the purpose of gathering relevant data to assist Lexus in making an informed decision on how to proceed with certified pre-owned vehicles.

\section{Procedures}

You will be asked to complete a survey concerning certified pre-owned vehicles. You will also be asked to provide your age, gender, and occupation. This survey will take approximately ten minutes. The data collected will remain anonymous and secure. Your participation is voluntary.

\section{Potential Risks}

- There are no known or anticipated risks to you by participating in this research.

- A further risk that should be identified is that the time inconvenience taken to complete this questionnaire may be problematic.

Potential Benefits 


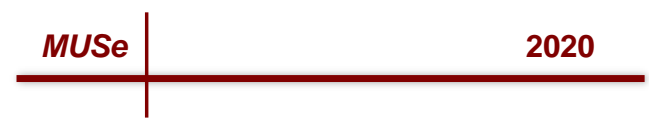

This project will provide insight to the perceived value of certified pre-owned vehicles. Our study will include the consumer's perception of certified versus non-certified, brand awareness, what adds value, and how much they are willing to spend on a used vehicle. This will provide benefits to Lexus on how to set up a new certification point process that will most effectively engage consumers. We anticipate these interviews will provide information on what adds value when consumers are looking to purchase a used vehicle which will further assist Lexus.

\section{Confidentiality/Anonymity}

- We will not collect any identifying information in the survey.

\section{Right to withdraw}

- Your participation is voluntary, and you can answer only those questions that you are comfortable with.

- You may withdraw from the research project for any reason, at any time, without explanation or penalty of any sort.

- Whether you chose to participate or not will have no effect on your class standing or how you will be treated.

- Should you wish to withdraw, there will be no penalties associated with it.

Follow up: If you are interested in the results, please contact for a copy of the report once it is completed.

\section{Storage of data}

- All data will be stored on the work computer of the principal investigator, which is password protected. Room 5-252E, CCC, MacEwan University.

- When the data is no longer required, the data will be destroyed.

\section{Questions or Concerns about Ethical Conduct:}


This project has been approved on ethical grounds by the MacEwan University Research Ethics Board on October 2, 2015. Any questions regarding your rights as a participant may be addressed to the Board at 780-633-3274 or REB@macewan.ca).

Project Title: Lexus of Edmonton Certified Pre-Owned Vehicle Survey 1. Do you consent to participating in this survey, based on the above information? *

$$
\square \text { पes } \square \text { No }
$$

2. How likely are you to purchase a pre-owned vehicle over a new vehicle?

$$
\begin{aligned}
& \square \text { Very Unlikely } \\
& \square \text { Unlikely } \\
& \square \text { Somewhat Likely } \\
& \square \text { Likely } \\
& \square \text { Very Likely }
\end{aligned}
$$

3. Do you know what a certified pre-owned vehicle is?

口Yes $\square$ No

4. Would you prefer to purchase a certified pre-owned vehicle over a non-certified pre-owned vehicle?

$\square$ Yes $\square$ No

5. If you found a pre-owned vehicle that met your specifications, how much more would you be willing to spend on a certified over a non-certified?
$\square$ Under $\$ 999$
$\square \$ 1000-\$ 1999$
$\square \$ 2000-$
$\$ 2999$
$\square \$ 3000$ - \$3999
$\square \$ 4000+$
$\square$ I would not spend more on a
certified pre-owned vehicle
$\square$ I would not purchase a pre-owned vehicle

6. If you found a pre-owned vehicle that met your specifications, how much are you willing to spend overall? 


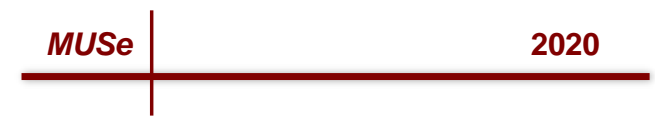

$\square$ Below $\$ 10,000 \quad \square \$ 10,000-\$ 15,000$

$\square \$ 15,001-\$ 20,000$

$\square$ Over $\$ 20,000 \quad \square$ I would not purchase a pre-owned vehicle

7. What would you like to see included in a certified pre-owned vehicle? (choose your top FIVE only)

$\square$ Less than 80,000 kilometers

$\square$ Made within the last 7 years

$\square 4$ months/40,000km Powertrain and Roadside Assistance coverage

$\square$ Full tank of gas

$\square$ Complimentary oil \& filter service

$\square$ Five (5) day $/ 1500 \mathrm{~km}$ (whichever comes first) grace period for swap or trade

$\square$ CarProof verification* includes accident report, recall, liens, etc.

$\square$ One-year end-to-end warranty

$\square$ Enrollment with dealership exclusives, including private events, free rentals, and VIP services

8. Are there any other options you would like to see included in a certified pre-owned vehicle that were not stated above?

$\square$ Yes $\quad$ No

(If yes) Please list the options you would like to see included in a certified pre-owned vehicle (Blank)

9. When looking at vehicle advertisements, which of the following most appeals to you?

$\square 131$ point check certified pre-owned vehicle

$\square$ Lexus of Edmonton certified pre-owned vehicle

$\square 210$ point check certified pre-owned vehicle

$\square$ CarProof verification certified pre-owned vehicle

$\square$ None of these appeal to me 
10. What is your preferred method of contact for communication?

$\square$ Email

$\square$ Phone Call

$\square$ Text

11. Have you ever been to the Lexus of Edmonton Dealership?

$\square$ Yes $\quad \square$ No

(If yes) How would rate your satisfaction level of the Lexus of Edmonton customer service?

$\square$ Very Satisfied

$\square$ Somewhat Satisfied

$\square$ Satisfied

$\square$ Somewhat Dissatisfied

$\square$ Very Dissatisfied

12. On a scale of 1 to 5 , with 1 being 'no trust' and 5 being 'complete trust' how would you rate your trust in Lexus of Edmonton in selling you a used vehicle?

13. What is your gender?
$\square$ Male
$\square$ Female
$\square$ Prefer not to answer

14. What is your current age?

$\square 25$ years or younger

$\square 26$ years or older

15. What is your current occupation?

$\begin{array}{ccccccc}\begin{array}{c}\text { No, I do not } \\ \text { trust }\end{array} & 1 & 2 & 3 & 4 & 5 & \\ \begin{array}{c}\text { dealerships at } \\ \text { all }\end{array} & & 0 & \bigcirc & \bigcirc & \bigcirc & \begin{array}{c}\text { Yes, I trust } \\ \text { them } \\ \text { completely }\end{array} \\ \end{array}$


$\square$ Part time

$\square$ Full time

$\square$ Student

$\square$ Retiree

$\square$ Unemployed 